\title{
LOS SISTEMAS CONTABLES ADMINISTRATIVOS: HACIA LA CONFORMACIÓN DE UN PROGRAMA DE INVESTIGACIÓN DESDE LA TEORÍA DE LA ESTRUCTURACIÓN*
}

\author{
CLAUDIA BARRIOS ALVAREZ" \& YAISMIR ADRIANA RIVERA ARRUBLA"** \\ PONTIFICIA UNIVERSIDAD JAVERIANA (CALI)
}

Recibido/ Received/ Recebido: 26/02/2010 - Aceptado/ Accepted/ Aprovado: 04/10/2010

\begin{abstract}
Resumen
El propósito del artículo es desarrollar un marco analítico vinculando la teoría de la estructuración de Giddens al análisis de los Sistemas Contables Administrativos y proponer a partir de este marco, un posible programa de investigación para el desarrollo de la contabilidad administrativa desde la perspectiva de análisis de las ciencias sociales. Así, se presenta primero, una síntesis de las categorías analíticas fundamentales de la teoría de la estructuración. Luego, se presenta una caracterización del contexto social en el cual es posible comprender la unidad de análisis propuesta, posteriormente, se sintetizan algunos trabajos de autores relacionados con la corriente críticointerpretativa alrededor de este tema. Finalmente, esta investigación plantea nuevas preguntas e hipótesis que podrían trabajarse en futuras investigaciones. Una primera hipótesis a comprobar, sería la existencia de la teoría de la estructuración como un posible programa de investigación para el desarrollo teórico de la contabilidad administrativa.
\end{abstract}

Palabras clave: teoría de la estructuración, sistemas de información administrativa, programa de investigación, contabilidad administrativa.

\section{ADMINISTRATIVE ACCOUNTING SYSTEMS: A REFLECTION FROM STRUCTURATION THEORY}

\begin{abstract}
The purpose of the article is to develop an analytical framework linking Giddens' structuration theory to Administrative Accounting Systems analysis and propose since this framework, a possible research program for administrative accountability development from social science analysis perspective. Thus, first a synthesis of fundamental analytical categories of structuration theory is presented. Then, the characterization of social context, in which it is possible to understand the
\end{abstract}

\footnotetext{
El presente trabajo hace parte del proyecto de investigación: "Papel que cumplen los Sistemas Contables Administrativos en el ordenamiento espacio-temporal de las prácticas sociales en las organizaciones modernas", el cual se deriva de la tesis de Maestría en Ciencias de la Organización de la Universidad del Valle, proyecto en el que las autores vinculan la teoría de la estructuración de Giddens al análisis de los sistemas contables administrativas en las organizaciones modernas. Este proyecto está inscrito bajo el código RII-277 en la Oficina de Investigación, Desarrollo e Innovación de la Pontificia Universidad Javeriana Cali, a cargo del Grupo de investigación "Pensamiento y praxis contable".

" Docente investigadora de la Pontificia Universidad Javeriana Cali, coordinadora del grupo de investigación "Pensamiento y praxis contable" de la PUJ Cali. Contadora Pública y Magíster en Ciencias de la Organización de la Universidad del Valle. Correo electrónico: claudiabarrios@javerianacali.edu.co

*** Docente de la Universidad del Valle, miembro del grupo de investigación "Pensamiento y praxis contable" de la Pontificia Universidad Javeriana Cali. Contadora Pública y Magíster en Ciencias de la Organización de la Universidad del Valle. Correo electrónico: yais36@hotmail.com
} 
proposed analysis unit, next to that, some similar studies are synthesized related to critical-interpretative current. Finally, this research states new questions and hypothesis that can be developed in future researches. One first hypothesis to prove would be the existence of the structuration theory as a possible research program for theoretical development of administrative accountability.

Key words: Theory of structuration, Administrative Accounting Systems, Management Accounting Systems, research programme, management accounting.

\title{
OS SISTEMAS DE CONTABILIDADE ADMINISTRATIVA: UMA REFLEXÃO BASEADA NA TEORIA DA ESTRUTURAÇÃO
}

\begin{abstract}
Resumo
Neste artigo se desenvolve um quadro analítico que relaciona a teoria da estruturação de Giddens e a analise dos Sistemas Contáveis Administrativos. Baseado neste quadro propõe-se um programa de pesquisa para desenvolver a contabilidade administrativa com a perspectiva de analise das ciências sociais. Primeiro, apresenta-se uma síntese das categorias analíticas fundamentais da teoria da estruturação. Logo se caracteriza o contexto social no que a unidade de analise pode compreender-se. Depois se sintetizam trabalhos de autores relacionados com o movimento crítico-interpretativo sobre este tema. Finalmente, esta pesquisa aborda novos interrogantes e novas hipóteses que devem examinar-se em pesquisas futuras. A primeira hipótese seria que a teoria da estruturação é um possível programa de pesquisa para o desenvolvimento teórico da contabilidade administrativa.

Palavras-chave: Teoria da estruturação, SCA, programa de pesquisa, contabilidade administrativa.

Barrios, C. \& Rivera, Y. (2010) Los sistemas contables administrativos: hacia la conformación de un programa de investigación desde la teoría de la estructuración. En: Revista de la Facultad de Ciencias Económicas de la Universidad Militar Nueva Granada. rev.fac.cienc.econ., XVIII (2)
\end{abstract}

JEL: M40, M41, M54.

\section{Introducción}

En la investigación contable, el uso de la teoría de la estructuración de Anthony Giddens se presenta como un recurso metodológico y una forma de comprender las relaciones sociales que contribuye al análisis del papel que cumplen los Sistemas Contables Administrativos (en adelante SCA) en los cambios organizacionales y sociales. Son varios los autores que con sus desarrollos teóricos dan sustento a esta afirmación, entre ellos, Pedro Araujo Pinzón, quién resalta la importancia que tienen teorías como la del nuevo institucionalismo en su vertiente sociológica y la teoría de la estructuración como marcos de referencia para el estudio de los sistemas contables en el contexto organizacional:
“... El nuevo institucionalismo sociológico es especialmente útil como marco de referencia para analizar cómo las ideas y presunciones sobre los sistemas contables de particulares campos organizativos son interiorizadas o no por las organizaciones sujetas a las presiones ejercidas por dicho campo. Complementando a lo anterior, la teoría de la estructuración posibilita investigar cómo la contabilidad incorpora el contexto institucional, al mismo tiempo que ejerce una fuerte influencia-directa e indirectamente- sobre cómo dicho contexto afecta a las organizaciones y la propia contabilidad" (Araujo; 2003, 710). 
A pesar que la teoría de la estructuración ha sido fuertemente cuestionada por la distancia entre el debate teórico que propone y la dinámica de la investigación empírica en las ciencias sociales (Clark, 1990 citado en Lawrence et al, 1997, 666), ésta puede sensibilizar a los investigadores hacia la naturaleza social de la práctica de la contabilidad, y en consecuencia, al examen detenido del papel que los SCA juegan en las organizaciones modernas.

El uso de la teoría de la estructuración permite de una parte, ubicar los SCA en el conjunto de las prácticas sociales, dimensionando cómo los contadores y la contabilidad como saber, están implicados en los cambios sociales y organizacionales, y a la vez, analizar las estructuras que se manifiestan a partir de las propiedades estructurales de los SCA estiradas en un tiempo y espacio en las organizaciones modernas, distinto a las condiciones espaciotemporales en las que fueron creadas.

La teoría de la estructuración ofrece sin duda un amplio potencial en la tarea de exploración que compete a la investigación contable-administrativa, interesada en una orientación sociológica. Este potencial está representado en las nuevas oportunidades que en este sentido brindaría la conformación de un programa de investigación para la contabilidad en materia teórica y metodológica, y por las posibilidades que su recategorización -para el caso específico de las organizaciones de América Latina y del país-, traería en la comprensión de los SCA. En efecto, al identificar las categorías analíticas que son aplicables a nuestro contexto, se podrían diseñar sistemas de administración y gestión contable más acordes a nuestra realidad que de esta manera respondan a las necesidades y dinámicas propias de nuestras organizaciones.

El presente artículo de reflexión se deriva de una investigación en la cual se construyó un marco analítico para estudiar el papel que cumplen los SCA en el ordenamiento espacio-temporal de las prácticas sociales en las organizaciones modernas, utilizando como marco de referencia las categorías conceptuales entregadas por Giddens en su teoría de la estructuración, analizada principalmente en La Constitución de la Sociedad (1998), Consecuencias de la Modernidad (1999), Las Nuevas Reglas del Método Socioló- gico (2001a) y Sociología (2001b). Estas categorías son contextualizadas en el marco de la modernidad -como experiencia vital con un especial ordenamiento de la vida organizacional y social- desde autores como Alfred Von Martin, Robert Nisbet, Marshall Berman, George Steiner, Norbert Elías, Fernando Cruz Kronfly y el mismo Anthony Giddens. Aunque estos desarrollos no son incluidos en el presente artículo, por razones de extensión y delimitación, sí constituyen la base desde la cual se propone la teoría de la estructuración vinculada al estudio de los SCA como un posible programa de investigación para el desarrollo de la contabilidad administrativa.

Reconociendo la importancia del desarrollo de un programa de investigación para la contabilidad administrativa que vincule la teoría de la estructuración al análisis de los SCA en esta investigación, se delinea aquí su posible conformación. En primera instancia, se presenta una síntesis de las categorías analíticas fundamentales de la teoría de la estructuración, luego se presenta la contextualización de la propuesta, posteriormente se hace una síntesis de cómo ha sido vinculada la teoría al estudio de la contabilidad administrativa y finalmente se presentan nuevas preguntas e hipótesis que podrían trabajarse en futuras investigaciones.

\section{Enfoque teórico}

\subsection{La Teoría de la estructuración}

La investigación surge y es desarrollada a partir del análisis de la teoría de la estructuración de Giddens. Esta teoría examina el modo en que las prácticas sociales son organizadas espacio-temporalmente, y cómo éstas se encuentran orientadas por unas estructuras que a su vez se manifiestan y actualizan en las acciones de agentes humanos entendidos.

Para aclarar lo que Giddens entiende por "estructuración" es necesario sintetizar algunos de los principales conceptos por él abordados al sentar las bases para su teoría.

\subsection{Estructura}

La siguiente es la acepción básica de estructura, en el contexto del análisis social: 
Estructura denota, entonces, en análisis social, las propiedades articuladoras que consienten la "ligazón" de un espacio-tiempo en sistemas sociales: las propiedades por las que se vuelve posible que prácticas sociales discerniblemente similares existan a lo largo de segmentos variables de tiempo y de espacio, y que presten a éstos una forma "sistémica" (Giddens, 1998, 54).

Siguiendo esta definición, las estructuras son propiedades que se articulan a través del anclaje espacio-temporal de prácticas similares en contextos diversos (segmentos variables de espacio-tiempo). La estructura conceptualizada de forma abstracta, reúne dos aspectos de reglas: elementos normativos y códigos de significación (coordinación de la actividad de agentes humanos), al tiempo que dos clases de recursos: de autoridad y de asignación (control sobre productos materiales o aspectos del mundo material) (Giddens, 1998, 32).

\subsection{Agencia}

En su concepción de agencia, Giddens parte del concepto de agente humano como un actor entendido, es decir, que todos los seres humanos, como actores sociales, son "expertos", en el sentido de poseer una conciencia práctica sobre el saber que poseen y que aplican en la producción y reproducción de encuentros sociales cotidianos (Giddens, 1998:58). Vale decir que el agente al que hace referencia su teoría es un ser humano apto para "obrar de otro modo", capaz de elegir entre intervenir en el mundo o dejar de hacerlo; capaz de producir una diferencia, capaz entonces de reservar para sí el ejercicio de algún tipo de poder. Agencia es entonces sinónimo de acción en cuanto a la posibilidad que se concede al agente, desde esta teoría, de ser portador de una aptitud de transformación.

En su obra, La constitución de la sociedad, Giddens aboga por una teoría en la que dialoguen agencia y estructura, entendiendo que si bien es necesario superar la visión subjetivista en teoría social en pro de la preeminencia de las estructuras defendidas por una ciencia social objetivista, tal propósito no puede redundar en que se pierda el significado del sujeto, ni mucho menos que el admitir el llamado a un descentramiento del sujeto, represente "la evaporación de la subjetividad en un vacío universo de signos" (Giddens, 1998:23). La teoría de la estructuración, concibe y reconceptualiza el lugar que ocupan tanto estructura como agencia, situando a las prácticas sociales inmersas en un espacio-tiempo, en la raíz misma de la constitución de la sociedad.

\subsection{Dualidad de Estructura}

Estructura y agencia forman parte de un mismo binomio indisoluble en esta teoría: "estructura no existe con independencia del saber que los agentes poseen sobre lo que hacen en su actividad cotidiana" (Giddens, 1998:62). Esta relación que denota fenómenos dependientes, en tanto los individuos tienen la posibilidad de actuar voluntariamente, enmarcados en unas estructuras las cuáles a la vez son creadas y recreadas en la interacción social, es denominada por Giddens como dualidad de estructura, concepto básico en la comprensión de su teoría de la estructuración.

La dualidad de estructura expresa la coexistencia de dos sentidos: por una parte, estructura, en su caracterización abstracta, como conjunto de reglas y recursos, está por fuera del espacio y tiempo, marcada además por una "ausencia de sujeto"; y por otra, estructura, que como elemento implícito en los sistemas sociales, remiten a la constitución de agentes situados, a las actividades reproducidas por un tiempo y un espacio en diversos contextos de acción (Giddens, 1998,61).

A través de esta dualidad, Giddens combina agencia y estructura, las cuales, lejos de corresponder a teorías mutuamente excluyentes, forman la base misma de la constitución de la sociedad y la explicación al comportamiento de los agentes.

Esta dualidad fundamenta la continuidad en la reproducción social a través del espacio-tiempo y parte de la capacidad de los agentes de registrar reflexivamente la duración de sus acciones. Sin embargo, dada la limitada capacidad de los agentes, siempre habrá lugar a que se gesten condiciones inadvertidas para la acción, ocasionadas por consecuencias 
no buscadas que superan las posibilidades de dominio absoluto. Esto, porque a pesar de que la historia está sustentada en actividades intencionales escapa, como un todo, a la posibilidad de ser un proyecto encaminado a responder a una teleología que direccione su trayectoria. No obstante, los seres humanos incluyen en la continuidad de sus actos el afán por someter su historia a su propio modelamiento.

\subsection{Dimensiones de estructuración}

La definición de estructura como "reglas y recursos con implicación recursiva en una reproducción social" (Giddens, 1998:32), contempla la división de dos especies de reglas: las reglas que atienden a los modos de significar o constitución de sentido, y a las sanciones normativas. Los recursos por su parte, hacen referencia al poder. A partir de esta caracterización puede llegarse al concepto de "dimensiones rectoras" de la dualidad de estructura.

Las dimensiones rectoras son tres, y corresponden a las estructuras de significación, legitimación y dominación. Las dos primeras (significación y legitimación) están constituidas por el tipo de elementos a los que Giddens atribuye el sentido de reglas; mientras la última, dominación, atañe a los recursos, sean éstos de autoridad o de asignación. Es importante anotar, como lo aclara Giddens, que cada una de estas tres dimensiones, sólo es separable analíticamente de las otras para fines explicativos.

Una vez establecidas estas tres dimensiones, éstas son precisadas por las "modalidades de estructuración", que refieren los rasgos estructurales utilizados por los actores en la reproducción de sistemas de interacción y la reconstitución de sus propiedades estructurales. Dichas modalidades son: los esquemas de comprensión, incluidos en las reglas relativas a estructuras de significación; las normas, como reglas referentes a estructuras de legitimación y las facilidades, entendidas como los recursos comprendidos en estructuras de dominación.
El nivel final de análisis para comprender la estratificación en que las estructuras son concebidas, está conformado por los aspectos de contextualidad de la acción, a los que Giddens denomina "interacciones" o formas de interacción. Las interacciones comportan la comunicación en el nivel de significación; la norma, en cuanto a las estructuras de legitimación, y el poder en el contexto de la dominación. Así, estructura, modalidad e interacción son los tres tipos de forma de institución a los que Giddens alude para sintetizar su teoría.

\subsection{Sistema Social}

Los sistemas sociales llevan implícita siempre, y de manera recursiva, una estructura. Estos sistemas comprenden las actividades situadas de agentes humanos, reproducidas en un tiempo y un espacio. Todo sistema social se fundamenta en las "actividades inteligentes de actores situados que aplican reglas y recursos en la diversidad de contextos de acción" (Giddens, 1998:61), actividades producidas y reproducidas en una interacción social.

Más que estructuras, los sistemas sociales presentan propiedades estructurales, en cuanto corresponden al orden virtual, el cual permite la reproducción de prácticas sociales rutinizadas; no obstante, estas últimas cobran una configuración con manifiesta presencia espacio-temporal en cuanto a su rol actualizador y como "huellas mnémicas" ${ }^{1}$ que orientan la conducta de agentes humanos entendidos.

\subsection{Desanclaje}

El concepto de desanclaje remite a la separación y posterior recombinación (reanclaje) del espacio y el tiempo, lo que hace posible una "precisa regionalización de la vida social" (Giddens, 1999, 28). El desanclaje, atinente a la separación entre el tiempo y el espacio, es un punto clave para caracterizar la discontinuidad existente entre las culturas tradicionales y el modo de vida moderno. Bajo el orden de la tradición existe una coordinación entre el tiempo y el espacio,

1 El término "huellas mnémicas" hace referencia a aquellas huellas registradas en la memoria que posibilitan la recordación posterior. Giddens (1998) considera que son la base del entendimiento humano y que como las estructuras, sólo se actualizan en la acción. 
es así como, dependiendo del tipo de actividad que realizaran las personas, estas daban cuenta de la hora del día. Las convenciones socio-espaciales anclaban tiempo y espacio, de manera que el cuándo (tiempo) estaba ligado al dónde (espacio y lugar).

\subsection{Rutina y rutinización}

La rutina es "un elemento básico de la actividad social cotidiana" La acepción de cotidianidad es entendida como el carácter rutinizado que corresponde a una vida extendida a través de un espacio-tiempo. La rutina es el fundamento material de la naturaleza recursiva de la vida social, y esta última, a su vez, comprende el carácter que poseen las propiedades estructuradas de la actividad social para ser recreadas continuamente a partir de los recursos que las constituyen. (Giddens, 1998:24), la rutina permite integrar rasgos tanto espaciales como temporales de encuentros sociales en procesos de constitución de sentido (Giddens, 1998: 65).

La recursividad de la vida social está asociada al entendimiento de que no son los actores quienes dan nacimiento a todas las actividades humanas de tipo social, sino que éstos las recrean continuamente a través de los mismos medios que les son dados para expresarse en tanto actores. La rutinización es la inclusión de la rutina como parte inherente de la vida social cotidiana, es un recurso que da fijeza a la acción humana, al obrar de los agentes. Giddens (1998:24) sostiene que "una rutinización es vital para los mecanismos psicológicos que sustentan un sentimiento de confianza o de seguridad ontológica durante las actividades diarias de la vida social".

\subsection{Seguridad ontológica}

La seguridad ontológica es un sentimiento de autonomía sobre el gobierno corporal dentro de rutinas predecibles. (Giddens, 1998:85), esta seguridad descansa y tiene soporte en la vida cotidiana ordinaria, es la posibilidad del actor de hacer de su cuerpo el lugar del propio-ser actuante, de tomar postura en el orden que le corresponde.

... La "seguridad ontológica" del ser humano hace referencia a la confianza que la ma- yor parte de los seres humanos tenemos en la continuidad de nuestra identidad y en la continuidad de nuestros entornos sociales y naturales de acción. Es decir, el individuo tiene la experiencia del "sí mismo" en relación a un mundo de personas y de objetos organizados simbólicamente, a través de la confianza básica (Trust, Vertrauen). (Giddens, 1990 citado en Beriain, 1996:26).

Aunque la seguridad ontológica signifique un elemento aparentemente inquebrantable y permanente para la construcción y el mantenimiento de la vida social cotidiana, para la continuidad de las rutinas, de las instituciones, de los sistemas sociales, y finalmente de la sociedad, existen no obstante, "situaciones críticas" en las que el individuo y/o de manera agregada, la comunidad, o incluso la humanidad entera, pueden ver amenazada la certidumbre en la que reposa la continuidad de sus rutinas, instituciones y formas de vida. Dichas situaciones críticas son indicadores de la llegada de circunstancias de inseguridad ontológica, las cuales señalan la amenaza a la futuridad de la duración de la vida social y la posibilidad de mantener el dominio sobre el gobierno del desenvolvimiento de la vida cotidiana.

\subsection{Encuentros}

"Los encuentros son el hilo conductor de una interacción social, la sucesión de compromisos con otros, ordenada dentro del ciclo de actividad diaria" (Giddens, 1990 citado en Beriain, 1996:106). Estos son "fenómenos secuenciados" que dan forma a la vida cotidiana mediante la rutinización, configurando a su vez el todo de la reproducción social a partir de la conexión entre el encuentro fugaz y la institucionalidad.

Los encuentros suponen tanto para su producción, como para su mantenimiento y reproducción, una serie de habilidades que sólo el agente apto sostiene. Dichas habilidades son entre otras: el gobierno reflexivo del cuerpo, el empleo de tacto y respeto como condiciones para la formación de la confianza que todo encuentro precisa, y la observancia de toda una serie de destrezas necesarias en una interacción y su reproducción. 


\section{Contextualización de la unidad de análisis}

El estudio del ordenamiento de las prácticas sociales a partir de los SCA constituye un aporte significativo en la tarea de comprender cómo las organizaciones afrontan actualmente el ambiente de constante riesgo e incertidumbre generado por las dinámicas de la modernidad. En efecto, el impacto global y el dinamismo de las instituciones modernas marcan una diferenciación radical frente al funcionamiento de las instituciones tradicionales. La forma y el ritmo en que los individuos viven el espacio-tiempo, también se manifiestan de manera muy distinta en el orden social moderno.

Bajo el orden de la tradición, en el mundo premoderno, las formas de calcular el tiempo se encontraban estrechamente vinculadas a la noción de espacio, las personas sabían de las horas en la medida en que podían referirse a otros indicadores socio-espaciales; el "cuándo" estaba estrechamente ligado al "dónde", anclando así tiempo y espacio. En el mundo moderno, el invento del reloj mecánico a fines del siglo XVIII y su amplia socialización, hacen que las personas den cuenta del tiempo -como tiempo uniforme y estandarizado mundialmente- sin referencia al espacio, también se produce la homologación mundial de los calendarios. Con el reloj se expresa el tiempo de manera uniforme, esto permite por ejemplo, designar de manera precisa "zonas" del día como la que corresponde a "la jornada laboral", en la cual el control sobre el tiempo posibilita un control sobre el espacio. El emparejamiento de la uniformidad de la medida del tiempo con la uniformidad en la organización social del mismo, rompe la conexión entre tiempo y espacio (Giddens, 1999:29), que vinculaba la noción de tiempo con el espacio donde eran realizadas las actividades.

El dinamismo (ritmo y ámbito de cambio) de la modernidad $^{2}$ proviene entonces del desanclaje de los sistemas sociales, concepto que como ya se señaló, remite a la separación y posterior recombinación (reanclaje) del espacio y el tiempo, posibilitando, tal como lo afirma Giddens, una precisa "regionalización" de la vida social, un ordenamiento y reordenamiento de las relaciones sociales (Giddens, 1999: 28). La separación del tiempo y el espacio se presenta como una condición inicial en el proceso de desanclaje, en tanto actividades sociales que antes se encontraban estrechamente conectadas a contextos de presencia particulares, hoy se pueden realizar por fuera de ellos, en la dinámica de las instituciones modernas y las organizaciones racionalizadas.

En los modos de vida tradicionales las personas tenían encuentros cara-a-cara, relaciones de copresencia. La modernidad ha permitido que los espacios sean redefinidos por múltiples lugares, dado que los individuos no requieren encuentros de mutua presencia para que las actividades de unos, ubicados en un determinado lugar, influyan en el espacio de otros, situados en un lugar distante.

Giddens sostiene que el dinamismo y reordenamiento de las relaciones sociales en la modernidad es posible entenderlos a partir del análisis de cómo se constituye la sociedad. El tipo de sociedad objeto de estudio de Giddens es la sociedad moderna, caracterizada por los Estados-nación; "sistemas perfectamente delimitados que poseen una unidad interna propia" (1999:25), cuyas organizaciones son diferentes a las formas de relación presentes en las sociedades tribales. En este mismo sentido, A este respecto, Renate Mayntz, tiene una mirada sobre las sociedades que confluye con la tesis en mención. Según la autora, las sociedades se distinguen de las comunidades por la existencia de organizaciones, las cuales regularizan el orden social bajo las exigencias de racionalidad contraídas por la modernidad. Mayntz (1972) reconoce tal papel a las organizaciones en su estructuración, y por tal razón acuña el término de "sociedad organizada", para explicar la importancia de las organizaciones en ésta, señalando que, a diferencia de lo ocurrido en el orden tradicional, gran parte de la vida social de los individuos está ordenada a través de las organizaciones.

2 ... La noción de "modernidad" se refiere a los modos de vida u organización social que surgieron en Europa desde alrededor del siglo XVII en adelante y cuya influencia, posteriormente, los han convertido en más o menos mundiales" (Giddens, 1999:15). 
Las organizaciones modernas han desbordado actualmente los límites de los Estados-nación, convirtiéndose en la principal forma de producción del capitalismo contemporáneo. En el caso de las grandes corporaciones, éstas pueden generar sus productos en distintos países, haciendo que sea más difícil su control por parte de un Estado particular. El capitalismo contemporáneo y su lógica de producción y consumo ha impactado significativamente a las sociedades a nivel mundial, fenómeno posibilitado por la infraestructura tecnológica de comunicación $e$ información que hoy permite una interconexión nunca antes vivida.

Para enfrentar el fuerte dinamismo del impacto global del capitalismo contemporáneo y ordenar las actividades diarias de las organizaciones, se han diseñado sistemas de interacción para recombinar (reanclar) espacio-temporalmente las relaciones sociales. Ejemplo de estos sistemas son los (SCA, los cuales están involucrados en la producción y reproducción de la vida organizacional, constituyendo e influenciando los procesos relacionados con gran parte de la información producida en las organizaciones; información que tiene un valor fundamental en un mercado global siempre en expansión y en el que compiten las organizaciones modernas.

Los SCA, son sistemas de interacción social, caracterizados por un espacio-tiempo contextualizado por límites físicos y simbólicos, que permiten su demarcación y diferenciación de otros escenarios comunes para el desarrollo de la vida social. También pueden ser entendidos, como portadores de la generación y el sostenimiento de instituciones tanto formales como informales, las que configuran la interacción social en las organizaciones y la emergencia de "estructuras estables" y procesos de cambio en las mismas. En el contexto organizacional, desde hace más de 20 de años, autores relacionados con la corriente crítico-interpretativa han tomado la teoría de la estructuración como marco interpretativo útil para estudiar los Management Accounting Systems $(\mathrm{MAS})^{3}$.

Los conceptos clave trabajados por Giddens, como: la dualidad de la estructura (estructura y agencia), las dimensiones de estructuración (significado, dominación y legitimación), las modalidades (esquemas de comprensión, facilidades y normas), el desanclaje (distanciamiento entre el espacio-tiempo), reanclaje (recombinación del tiempo y espacio), zonificación del tiempo y el espacio, han sido aplicados al estudio de los MAS, generando diversas formas de concebirlos.

Uno de los trabajos base para vincular la teoría de la estructuración al análisis de los MAS es el de Roberts y Scapens: Accounting Systems and Systems of Accountability-Understanding Accounting Practices In their Organizational Contexts publicado en 1985 y el cual se ha convertido, en referencia obligada para quienes han centrado sus investigaciones en esta temática.

3 En esta propuesta de investigación, la denominación "MAS" será usada en adelante para hacer referencia a los Management Accounting Systems, en consonancia con la sistematización de la literatura revisada en el estado del arte. El concepto SCA no necesariamente corresponde a las interpretaciones que sobre sistema contable y contabilidad administrativa han realizado los autores de la corriente crítico-interpretativa. Aunque un análisis detenido sobre la diferenciación conceptual entre los términos MAS y SCA desborda los límites del estado actual de la investigación, sí es posible precisar que en esta investigación se entiende por contabilidad administrativa tanto aquella que capta, registra, acumula y permite asignar valor a los diferentes procesos acaecidos en una organización, como la que provee los mecanismos para su autocontrol y evaluación. En el primer sentido la contabilidad permite determinar el costo de productos y servicios (contabilidad de costos), y en el segundo, genera al tiempo la base informativa para la planeación y el control de todas las actividades de la organización susceptibles de valuación monetaria, promoviendo y facilitando los procesos de toma de decisiones (contabilidad de gestión y contabilidad financiera), para la proyección y sostenibilidad tanto en el corto, como en el mediano y largo plazo de la organización. La idea de contabilidad administrativa que orienta esta investigación, junto con la construcción de la noción de SCA's, parte de la comprensión de que no sólo -y ni siquiera principalmente- la información resultante del proceso contable, expresada en los informes de tipo contable-financiero, sino más bien los procesos que se adelantan a diario y de manera reflexiva por los agentes del hacer contable en las organizaciones, constituyen el núcleo de interés a observar y a pensar en adelante en el estudio, diseño e implementación de sistemas contables. Este marco de análisis no será aplicado sólo en sistemas de información contable, sino en sistemas de interacción social permeados por procesos contables orientados a administrar y gestionar las prácticas cotidianas a través de la comprensión de las manifestaciones presentes en las diferentes rutinas y encuentros. 
Los autores para estudiar los sistemas contables elaboran una distinción inicial entre MAS y los sistemas de responsabilidad. Para ellos el concepto de MAS corresponde al conjunto abstracto de reglas y recursos usados por los individuos en sus contextos de interacción, y los sistemas de responsabilidad son el conjunto de prácticas institucionalizadas que representan el uso real de los MAS.

Los autores analizan cada una de las dimensiones de la estructura (de significado, legitimación y dominación) para comprender cómo la información contable está implicada en la formación y mantenimiento de los sistemas de responsabilidad en las organizaciones y a la vez cómo estos sistemas se expresan en las acciones exigidas a los individuos.

Esta concepción de los sistemas de responsabilidad como el conjunto de prácticas institucionalizadas y el análisis de los MAS desde las tres dimensiones de la estructura propuestas por Giddens ha sido ampliamente compartido por diferentes autores, tales como: Lawrence, M. Alam, D. Northcott \& T. Lowe (1997), quienes en su trabajo titulado: Accounting systems and systems of accountability in the New Zealand health sector, estudian desde la teoría de Giddens los modos institucionalizados de interactuar (estructuras de significación, legitimación y dominación) y los aplican a los procesos de reforma del sistema de salud de Nueva Zelanda, antes y después de su inicio, en julio de 1993.

En esta línea de pensamiento, también está inscrito el trabajo de Macintosh \& Scapens (1991), Management Accounting and Control Systems: a structuration theory-analysis. En este artículo los autores utilizan dos estudios de caso para identificar los procesos de estructuración en sus tres dimensiones. El primero, es la descripción detallada de Sloan, acerca del desarrollo de controles financieros en General Motors. El segundo, expone la evolución del MAS para la reparación de armas del Departamento de Defensa de los Estados Unidos a partir del trabajo de Ansari y Euske.
En el artículo de Macintosh \& Scapens, Structuration theory in management accounting (1990), también se analizan las dimensiones de dominación, legitimación y significado de las prácticas administrativas en el estudio de caso realizado por Covaleski y Dirsmith, sobre el sistema presupuestal de la Universidad de Wisconsin ${ }^{4}$.

Otro artículo referenciado frecuentemente es el de Burns y Scapens (2000), Conceptualizing management accounting change: an institutional framework" Management Accounting Research por los conceptos desarrollados a partir de la Teoría de la Estructuración. En este artículo los autores analizan desde un enfoque institucional cómo las prácticas y rutinas de la contabilidad administrativa hacen parte de un espectro más amplio de rutinas en la organización y cómo éstas entran a hacer parte de la manera como se hacen las cosas.

En ocasiones los autores definen la contabilidad administrativa y los MAS como rutinas potencialmente institucionalizadas (prácticas organizacionales). De acuerdo al esquema planteado por Giddens sobre los modos de estructuración (estructuras, modalidades e interacción), el planteamiento de los MAS de estos autores estaría ubicado en primera medida en el nivel de las modalidades puesto que consideran que los MAS median entre las propiedades estructurales, las instituciones y la acción. Sin embargo, siguientes sus propios conceptos, también entrarían en el nivel de interacción, pues definen los MAS como rutinas institucionalizables.

La distinción de las reglas y rutinas que componen los MAS cuando son conceptualizadas como modalidades utilizadas por los individuos en su interacción social y cuando son conceptualizadas como la práctica contable administrativa como tal, es trabajada por Englund \& Gerdin (2007) en su artículo, Structuration theory and mediating concepts: pitfalls and implications for management accounting research. En este artículo se retoman a autores como Burns \& Scapens para evidenciar las inciden-

4 Un aspecto trabajado frecuentemente en los artículos revisados es el de las dimensiones de la estructuración (significado, legitimación y dominación) con más énfasis que cuando el mismo Giddens lo plantea en la formulación de las bases de la teoría de la estructuración. 
cias metodológicas y teóricas en el desarrollo de la investigación contable-administrativa por el uso de diversas concepciones de los MAS.

Frente a los diferentes definiciones de los MAS y las diversas formas de incorporar los conceptos de Giddens a las investigaciones en la contabilidad administrativa, también se han generado fuertes debates entre autores cómo Macintosh, Scapens \& Boland. Este debate inicia cuando Boland escribe su artículo Accounting and the Interpretative Act (1993) en el cual realiza fuertes cuestionamientos a los planteamientos de Macintosh y Scapens, señalando que estos autores tienen una posición marcadamente estructuralista en el estudio de los MAS porque le otorgan un excesivo papel a la estructura en la creación de significados, ignorando el papel de la agencia en este proceso pese a la importancia que ésta tiene en la teoría de la estructuración de Giddens (Macintosh \& Scapens, 1996, 675) y como respuesta a estos cuestionamientos Macintosh \& Scapens (1996), publican el siguiente artículo: Structure and agency in management accounting: a response to Boland's interpretive act en el cual clarifican las posturas que han asumido en sus investigaciones y manifiestan que Boland distorsiona el significado de sus trabajos.

Frente a la réplica que realizan Macintosh \& Scapens del primer artículo de Boland (1996), éste último continúa el debate presentando su artículo Why shared meanings have no place in structuration theory: A reply to Scapens and Macintosh. Esta réplica de Boland, consiste en una aclaración de que no es la distinción entre el nivel del análisis (institucional o de la conducta estratégica), lo que diferencia la posición de unos y otros autores, sino la comprensión misma de la teoría de la estructuración. Según Boland, Macintosh \& Scapens, se ubican a sí mismos en el nivel del análisis institucional, sugiriendo que Boland se concentra en el de la conducta estratégica. No obstante, aclara que no es la referencia al nivel o al tipo de estudio aquello en lo que radica la dificultad central entre sus puntos de vista, sino al entendimiento y lugar dado en su interpretación de la teoría a los significados, creencias, intenciones y motivaciones compartidos por los agentes.
Esta distinción manifestada por Boland, es importante porque la interpretación dada por Macintosh y Scapens a los significados y valores compartidos tiene implicaciones tanto para la interpretación de informes contables, como para los MAS y agregadamente en la explicación de los procesos institucionales. Finalmente, es importante incluir en este apartado dos trabajos de autores que socializan sus resultados de investigaciones aplicadas, en las cuales utilizaron como marco de referencia los planteamientos de Giddens.

Busco, Riccaboni \& Scapens (2000), en su artículo titulado Management Accounting Systems and Organisational Culture: an Institutional Framework for Interpreting their Linkages and Processes of Change estudian la compañía italiana, Nuovo Pignone -NP- en el momento en que experimenta dos cambios importantes, su adquisición por parte de General Electric y la implementación de un nuevo programa de calidad basado en la medición, el Six-sigma.

Los autores estudian el papel jugado por la información contable y financiera en los procesos de cambio cultural de la compañía dada la relevancia que esta información tomó al constituir un lenguaje común no sólo para los contadores, sino para los mandos altos y medios de la empresa. La cultura de la medición fue incorporada en las prácticas cotidianas de los individuos.

Al estudiar el papel desempeñado por los MAS en la trasformaciones organizacionales y su interconexión con los cambios culturales, los autores plantean una asunción básica, y es que los MAS basados en la medición del desempeño y la responsabilidad tienen el potencial de estructurar el orden organizacional (Busco \& et al, 2000, 35), al representar un conjunto de reglas, roles y rutinas portadores de los valores organizacionales compartidos. Otro estudio de caso hallado en esta investigación y que está directamente relacionado con el estudio de los MAS, es el de Carmona, Ezzamel \& Gutiérrez, 2002), titulado The relationship between accounting and spatial practices in the factory.

En este artículo los autores estudian la relación existente entre las prácticas espaciales y las prácticas 
contables de una fábrica española productora de Tabaco, Royal Tobacco Factory (RTF), la cual es movida del centro a las afueras de la ciudad de Sevilla. En este estudio, los autores exploran las maneras como las prácticas contables forman y a la vez son condicionadas por las prácticas espaciales (Carmona, Ezzamel \& Gutierrez, 2002, 241). También exponen algunas maneras a través de las cuales el espacio puede ser demarcado por la contabilidad.

Los autores sostienen que las prácticas contables tienen la capacidad para funcionar como dispositivo de ordenamiento espacio-temporal (Carmona, Ezzamel \& Gutierrez, 2002, 239), y enlazadas con las prácticas espaciales, proveen el alcance para que los administradores refuercen la vigilancia sobre los empleados. En el estudio se evidenció la manera como los centros de costos crearon un espacio analítico contable útil para el control del espacio físico, permitiendo una cuantificación, codificación y evaluación del desempeño de los operarios en cada etapa de producción de la fábrica. Cada responsable de un centro de costos reportaba información contable detallada sobre las existencias de tabaco, las horas trabajadas y la ubicación espacial de cada operario.

Con el análisis de los artículos revisados en esta investigación, es posible dimensionar las posibilidades abiertas al campo de la investigación contable con la utilización de la teoría de la estructuración y otros conceptos desarrollados por Giddens, pues éstos permiten captar la naturaleza de la intervención de los SCA en las organizaciones modernas y también posibilitar aún más la investigación empírica, dado que es posible que al centrarse más en los aspectos sociológicos de la intervención de los SCA en el ordenamiento espacio-temporal de las prácticas sociales en las organizaciones modernas, sea menos difícil acceder a los archivos fuentes de investigación, pues distintos documentos (actas, cartas, informes, entre otros) o la observación directa de actividades dentro de las organizaciones pueden arrojar datos a estas investigaciones de tipo cualitativo. Desafortunadamente el poco desarrollo de la investigación empírica en contabilidad en nuestro contexto se debe en parte a la imposibilidad de acceder a la información contable básica para el desarrollo de los estudios de caso.

\section{Nuevas perspectivas de investigación en contabilidad administrativa: preguntas e hipótesis}

\subsection{Lateoría de la estructuración como fundamento teórico para el desarrollo de un posible programa de investigación en contabilidad administrativa}

La teoría de la estructuración puede ser considerada como un proyecto conceptual, idea extraída de la propia propuesta de Anthony Giddens, quien considera que la suya, más que una teoría, corresponde a un proyecto que atraviesa, tanto propósitos teóricos, como posibilidades de aplicación empírica.

Después de presentar el contexto desde el cual es posible analizar los SCA a partir de la teoría de la estructuración y entregar una breve síntesis de los trabajos que desde la corriente crítico interpretativa han vinculado esta teoría al análisis de los MAS, se presenta a la comunidad de investigadores interesados en el desarrollo de la investigación contable-administrativa desde una perspectiva sociológica, una nueva pregunta que se plantea a partir del presente trabajo: ¿qué elementos de la teoría de la estructuración podrían servir para la fundamentación de un programa de investigación para la contabilidad administrativa?. Esta pregunta se puede responder a manera de hipótesis, señalando la existencia de varios elementos conceptuales, que como la presencia de un núcleo firme, permitirían hablar de la teoría de la estructuración vinculada al estudio de los SCA, como un posible programa de investigación contable administrativa. Hipótesis que de ser acogida, daría paso a futuras investigaciones en disciplinas que discurren sobre lo organizacional y lo contable.

\subsection{Características de un programa de investi- gación desde la teoría de la estructuración}

Lakatos propone su metodología de los Programas de investigación científica con el ánimo de resolver algunas problemáticas no solucionadas por sus antecesores Karl Popper y Tomas Kuhn. Una de estas problemáticas hace referencia a cómo se construye y evoluciona la ciencia a través de la constitución de los Programas de investigación científica. 
Lakatos plantea que los programas de investigación científica representan la unidad descriptiva típica de los grandes logros científicos y de su construcción racional, orientados por "una heurística que incluye un conjunto de técnicas para la solución de problemas" (2007, p. 230). Esta unidad corresponde a una serie estructurada de teorías en desarrollo y no a una hipótesis planteada de manera aislada. El programa de investigación científica está constituido por "reglas metodológicas: algunas nos dicen las rutas de investigación que deben ser evitadas (heurística negativa), y otras, los caminos que deben seguirse (heurística positiva) (Lakatos, 2007, p. 65).

Los programas de investigación están constituidos por un núcleo firme que contiene los conceptos básicos de la teoría, considerados irrefutables por decisión metodológica de los defensores. La heurística negativa marcaría aquella ruta metodológica que impide que ese núcleo firme del programa sea cuestionado. Los científicos construyen cinturones protectores alrededor de ese núcleo firme. Este cinturón está compuesto de hipótesis auxiliares ad hoc útiles para la resolución de anomalías o hechos que el programa no puede explicar. Las contrastaciones o refutaciones afectan el cinturón protector del programa más no su núcleo firme, de esta manera las hipótesis auxiliares pueden ser ajustadas e incluso refutadas mientras el núcleo firme del programa permanece intacto (Lakatos, 2007, 66-69). El proceso de construcción del cinturón protector es lo que Lakatos denomina heurística positiva, la cual "consiste de un conjunto, parcialmente estructurado, de sugerencias o pistas sobre cómo cambiar y desarrollar las 'versiones refutables' del programa de investigación, sobre cómo modificar y complicar el cinturón protector 'refutable"” $(2007,69)$.

El trabajo que hace la comunidad científica para preservar el núcleo de la teoría, consiste en el ajuste y reajuste de las hipótesis auxiliares que componen el cinturón protector, y en el que tiene lugar la escogencia de los problemas de los que se han de encargar. El programa de investigación se considerará progresivo mientras sea capaz de producir nuevos hechos e hipótesis auxiliares. Esto, hasta el momento en que las anomalías se resistan a desaparecer, tras la disminución de la eficacia de la heurística po- sitiva, momento en el cual se considera que el programa degenera y empieza a estancarse.

Los científicos adheridos a un programa, sólo han de abandonarlo una vez el mismo deje de mostrarse promisorio y un nuevo programa se muestre capaz de mayor contenido empírico y prometa una mayor capacidad de explicación y predicción.

La idea del programa de investigación, es afín, en varios aspectos al concepto de paradigma o matriz disciplinaria que desarrolla Thomas Kuhn en su obra, principalmente en La Estructura de las revoluciones científicas. Ambos, tanto los programas de investigación, como los paradigmas, parten del reconocimiento de la necesidad de acudir a la historia, para revisar la manera en que las teorías han sido construidas y mantenidas por las comunidades científicas, entendiendo que la ciencia es un tipo de conocimiento que aunque busca estabilidad para su desarrollo, está indefectiblemente abierto al cambio para asegurar su evolución.

Los cambios revolucionarios en el período de ciencia normal implican la emergencia de un nuevo paradigma inconmensurable con relación a su predecesor, el cual trae su propios criterios, "el nuevo paradigma trae consigo una racionalidad completamente nueva" (Lakatos, 2007, 120) es, lo que en términos de Kuhn se denomina como revoluciones científicas. El cambio de paradigma, implica una transformación de la ciencia hacia una nueva manera de ver el mundo, sustentada en el abandono del paradigma anterior. El cambio de un programa de investigación a otro, por su parte, indica que el programa vigente se apague; pero no descarta que el mismo pueda cobrar vigencia en el futuro al reavivarse el interés de la comunidad científica por sus planteamientos, lo cual parece mucho más próximo a la naturaleza de las ciencias sociales, provistas de mayor inestabilidad y posibilidades de regresión hacia formas anteriores de desarrollo en un camino que a la vez signifique evolución.

Por el concepto de paradigma, en cuanto a su afinidad al de programa de investigación, y su propiedad para señalar la guía que por sí mismo constituye para el trabajo científico, se entiende principalmente lo siguiente: 
Realizaciones científicas universalmente reconocidas que, durante cierto tiempo, proporcionan modelos de problemas y soluciones a una comunidad científica (Kuhn, 2000, 13).

... síntesis capaz de atraer a la mayoría de los profesionales de la generación siguiente... (Kuhn, 2000, 45).

... criterio para seleccionar problemas que, mientras se dé por sentado el paradigma, puede suponerse que tienen soluciones... (Kuhn, 2000, 71).

... fuente de coherencia para las tradiciones de investigación normal... (Kuhn, 2000, 79).

Las amplias críticas presentadas al concepto de paradigma, especialmente por parte de Margaret Masterman $^{5}$ hacen que Kuhn decida acudir al uso de "matriz disciplinaria"6 para reemplazar el término paradigma, a fin de establecer con mayor precisión qué comparten los miembros de una comunidad científica. A juicio de Kuhn, "matriz disciplinaria" hace referencia al conjunto de elementos ordenados de distinta índole (constelación de compromisos del grupo), del que disponen los practicantes de una disciplina particular.

Las siguientes son las principales clases de componentes de una matriz disciplinar, las cuales integran los elementos necesarios para el ejercicio de la ciencia en el seno de una comunidad científica:

1. Generalizaciones simbólicas: Éstas corresponden a los componentes formales, o fácilmente formalizables.

2. Creencias en modelos particulares con inclusión de una variedad relativamente heurística: Proveen a la comuni- dad científica de las analogías y metáforas permisibles, ayudando a determinar lo que será aceptado como explicación y solución de problemas y a determinar los enigmas no resueltos.

3. Valores: Se refieren a los valores compartidos en todo momento por la comunidad científica, cuya importancia se realza en el momento de identificar y afrontar una crisis.

4. Ejemplares: Referentes a las soluciones concretas de problemas que dan a la comunidad una estructura más fina de la ciencia. (Kuhn, 2000: 278-287).

Llegados a este punto, es preciso aclarar que así se haga uso del término "programa de investigación", o el de "paradigma" para designar la vigencia y alcance de la teoría de la estructuración como teoría sociológica capaz de atraer y guiar a la comunidad científica para el desarrollo y práctica de las ciencias sociales, ninguno de los dos fue inicialmente pensado para el estudio de éstas. Kuhn, en su posdata de 1969 a la Estructura de las Revoluciones Científicas, hace saber que aunque agradece la adhesión a sus teorías por parte de muchos lectores de disciplinas distintas a la ciencia, su trabajo fue pensado y elaborado desde y para la ciencia, y más específicamente para la física. Igualmente, el trabajo de Lakatos, es producto de una revisión refinada del falsacionalismo popperiano, la cual se desprende principalmente del análisis del desarrollo de la física newtoniana.

No obstante, hecha esta precisión, y guardando las distancias que de hecho existen entre los conceptos de paradigma y programa de investigación, ambos y particularmente este último, podrían aportar un marco propicio para el desarrollo de lo que Giddens denomina como su proyecto conceptual, al referirse a la teoría de la estructuración y la tarea que ésta tiene en las ciencias sociales. La teoría de la

\footnotetext{
Para ampliar más al respecto veáse The nature of a Paradigma en Growth of Knowledge, de Margaret Masterman.

6 El reemplazo del término paradigma, por el de matriz disciplinaria, es al parecer provisional, entre tanto no se alcance que el concepto de paradigma quede libre de sus actuales implicaciones. En cuanto a por qué acudir a matriz disciplinaria, Kuhn explica que: "disciplinaria' porque se refiere a la posesión común de quienes practican una disciplina particular, 'matriz' porque está compuesta por elementos ordenados de varias índoles”. KUHN. Posdata de 1969 (2000, 279-280).
} 
estructuración, entendida como programa de investigación o paradigma, será entonces un modelo que aporta al practicante de una disciplina científica social, en estado normal, el marco aceptado por la comunidad científica de teorías, valores, problemas y soluciones esperados, además de conllevar un sentido y una visión acerca del mundo y los referentes que estudia: las generalizaciones simbólicas, las guías, herramientas, y en general, las bases para trabajar dentro de una tradición de investigación de ciencia social en un contexto histórico. Todo esto, en un estado de cohesión y coherencia interna de carácter falible, que garantiza la revolución y asegura la supervivencia del ciclo de desarrollo de una disciplina científica con cierto grado de madurez.

Con el propósito de establecer el alcance posible que la teoría de la estructuración pueda tener como programa de investigación y aun como paradigma, en el campo de la contabilidad administrativa, es importante integrar el concepto de Kuhn, de matriz disciplinaria, dada sus importantes implicaciones epistemológicas y metodológicas, con el fin de constituir lo que podríamos denominar un programa matricial de investigación, bajo el cual la comunidad académica contable pueda guiar sus investigaciones desde una perspectiva sociológica. Esta tarea se encuentra inmersa tanto en el debate filosófico que se propone el descubrimiento y/o formulación de generalizaciones teóricas, como en el trabajo empírico impulsado por la búsqueda del saber que los agentes construyen en la reproducción de sus prácticas cotidianas, saber este, que es contingente, y que otorga un carácter dinámico e inestable a la teoría sociológica.
La sistematización del pensamiento de Giddens, se constituiría en un verdadero programa de investigación para la contabilidad administrativa, el cual estaría provisto de un núcleo firme alrededor de las categorías centrales de su teoría: acción y estructura $^{7}$; las cuales se consolidan en el concepto de dualidad de la estructura ${ }^{8}$ y todo el aparataje conceptual que la sustenta. Dicho núcleo haría posible el funcionamiento de una heurística negativa.

De otra parte, el rastreo de las investigaciones en contabilidad administrativa a nivel teórico y aplicado basadas en la teoría de la estructuración, darían cuenta de la existencia efectiva de ésta como posible programa matricial de investigación. La revisión de los resultados de las investigaciones en este ámbito y las críticas posibles al programa, serían la materia prima para formular una heurística positiva, que contendría las hipótesis auxiliares ajustables que los investigadores incorporen e incluso inventen para protegerlo de aquellas refutaciones o anomalías que éste pudiera contener.

A continuación se presenta una agenda metodológica a tener en cuenta en el desarrollo del programa de investigación de la contabilidad administrativa.

\subsection{Propuesta de un itinerario metodológico apropiado para el programa matricial de in- vestigación en contabilidad administrativa}

Recapitulando algunos de los planteamientos hechos a través de la presente investigación, es necesario hacer énfasis en el amplio campo de acción que ofrece la teoría de la estructuración a propósito de la investi-

7 El reemplazo del término paradigma, por el de matriz disciplinaria, es al parecer provisional, entre tanto no se alcance que el concepto de paradigma quede libre de sus actuales implicaciones. En cuanto a por qué acudir a matriz disciplinaria, Kuhn explica que: "disciplinaria' porque se refiere a la posesión común de quienes practican una disciplina particular, 'matriz' porque está compuesta por elementos ordenados de varias índoles". KUHN. Posdata de 1969 (2000, 279-280).

8 Respecto al concepto de dualidad de la estructura (estructura y agencia), en el cual se considera, radica el núcleo central del programa de investigación de Giddens, es oportuno hacer aquí la siguiente ampliación sobre las razones que lo llevaron a elaborar este concepto: “... Lo hice para combatir dos tipos principales de dualismo. Uno es el que encontramos en perspectivas teóricas preexistentes. Sociologías comprensivas como las que examiné en Las nuevas reglas, según lo expresé en otra parte, son 'fuertes en acción pero débiles en estructura'. Para ellas, los seres humanos son agentes intencionales, conscientes de sí, que pueden aducir razones para lo que hacen; pero tienen pocos recursos para abordar cuestiones que en cambio, y con todo derecho, adquieren todo su relieve en los abordajes funcionalistas y estructurales: problemas de constricción, de poder y de organización social de vasta escala. Por su parte, los abordajes de este segundo grupo son "fuertes en estructura" pero "débiles en acción". Los agentes se presentan inertes e ineptos: juguete de fuerzas que los superan. (GIDDENS, A. Las nuevas reglas del método sociológico. op. cit. p. 14). 
gación contable-administrativa. Las ideas de Giddens, articuladas al interés de estudiar los procesos sociales configurados desde la contabilidad, entendida como esquema de comprensión, proveen elementos fundamentales para la conformación de un programa de investigación alrededor de esta temática. Ello representaría para el estudio de la contabilidad y los sistemas contables un avance significativo en materia teórica y metodológica, comprendiendo que la contabilidad podría ser desarrollada en el ámbito de las ciencias sociales, en tanto el saber contable como las prácticas asociadas a él, están inmersos en el conjunto de prácticas que estructuran las organizaciones y por ende la sociedad en general.

Pensar en la teoría de la estructuración como un marco de análisis para el estudio teórico y la construcción metodológica de los SCA, implica entre otras cosas, desplazar la mirada de lo que podría considerarse como la salida o producto de los sistemas contables: la información; centrando el interés ahora, sobre los procesos; y de esta manera desplazar simultáneamente el enfoque desde el paradigma de la utilidad, hacia el paradigma, o mejor, programa matricial de investigación estructuracionista; es decir, específicamente bajo esta nueva cosmovisión, la piedra de toque serían las prácticas sociales que se gestan cotidianamente en los sistemas de interacción social, como producto del hacer inteligente de actores situados espacio-temporalmente en contextos marcadamente de tipo contable-administrativo.

\subsection{Posibles indicios del programa de investi- gación estructuracionista vinculado al estudio de los SCA}

La idea de pensar la constitución de un programa de investigación de la teoría de la estructuración para los SCA, viene dada no sólo por las capacidades explicativas de la teoría sino también por la pertinencia de estudiar tanto la contabilidad como las prácticas contables, consideradas como parte de un contexto social más amplio en el cual se desarrollan, pertinencia que ya ha sido evidenciada por varios investigadores de la contabilidad administrativa.
En la búsqueda bibliográfica llevada a cabo en esta investigación, se encontró un artículo de Jones, Matthew R. \& Karsten, Helena (2008), titulado Review: Giddens's Structuration theory and information Systems research, en el cual se elabora un inventario de la aplicación de la teoría de la estructuración a la investigación de los sistemas de información en general. Los autores clasifican sus hallazgos en cuatro temáticas presentadas en la Tabla No. 1 .

Tabla 1. Resumen de la revisión de la teoría de la estructuración vinculada al estudio de los sistemas de información ${ }^{9}$

\begin{tabular}{|l|c|}
\hline \multicolumn{1}{|c|}{ Temática } & $\begin{array}{c}\text { No de trabajos } \\
\text { hallados }\end{array}$ \\
\hline $\begin{array}{l}\text { Aplicación de los conceptos estructu- } \\
\text { rales }\end{array}$ & 121 \\
\hline $\begin{array}{l}\text { Aplicación de conceptos de otros es- } \\
\text { critos de Giddens. }\end{array}$ & 33 \\
\hline $\begin{array}{l}\text { Desarrollo y aplicación de una versión } \\
\text { específica de la teoría de la estructu- } \\
\text { ración }\end{array}$ & 113 \\
\hline $\begin{array}{l}\text { Compromiso crítico con la teoría de la } \\
\text { estructuración }\end{array}$ & 67 \\
\hline Totalidad de artículos referenciados & 334 \\
\hline
\end{tabular}

En la tarea de revisar la existencia del programa matricial de investigación que vincule la teoría de la estructuración al estudio de los SCA, es posible partir del número significativo de trabajos referenciados en el artículo de Matthew \& Karsten (2008) para identificar cuáles están directamente relacionados con el programa de investigación, hallar las interconexiones entre los diferentes trabajos de los autores, sus posibles interlocuciones y la existencia misma de una comunidad científica comprometida con el estudio de los SCA desde la teoría de la estructuración.

Ahora bien, al cierre de esta investigación, se propone una agenda de trabajo como una posibilidad abierta para continuar el estudio acerca de cómo los SCA ordenan las prácticas sociales en las orga-

9 Matthew, R. \& Karsten, H. Review: Giddens's Structuration theory and information Systems research. En: MIS Quarterly Vol. 32 No. 1, 2008. 
nizaciones bajo el contexto moderno y a partir del programa de investigación estructuracionista:

\subsection{Escenarios posibles en el desarrollo del programa de investigación estructuracionista vinculado al estudio de los SCA}

En el camino de constituir la teoría de la estructuración como un programa de investigación para el estudio de los SCA, es necesario hacer una revisión de los trabajos elaborados alrededor de esta temática, evidenciando cuáles se orientan más hacia la conceptualización y cuáles han utilizado los marcos analíticos ya elaborados para su aplicación en estudios de caso específicos, desde los cuales también es posible conceptualizar teniendo en cuenta los resultados obtenidos.

Las conceptualizaciones pueden ser de distintos órdenes -primarios o secundarios- y también resultado de diferentes tipos de investigación (básica o aplicada) que pueden ser clasificadas de la siguiente forma:

\section{a) Conceptualizaciones generales sobre los}

SCA: En este nivel podrían precisarse las distintas formas mediantes las cuales los investigadores de la contabilidad administrativa han conceptualizado los sistemas contables y de ser posible identificar o construir un concepto que sea ampliamente compartido por la posible comunidad de investigadores de la contabilidad administrativa.

Siguiendo el marco propuesto por Giddens, se encuentran tres formas de institución: significación, dominación y legitimación, al mismo tiempo que el análisis sobre el tipo de estructura, de modalidad y de interacción, comprometidas en cada dimensión, sería posible clarificar el lugar que ocupa la contabilidad en este esquema conceptual e identificar las variadas formas bajo las cuales ha sido y puede ser conceptualizado este saber.

En estas conceptualizaciones generales, también podrían participar los contadores públicos con una amplia trayectoria en su ejercicio profesional, quienes conocerían desde su ex- periencia inmediata cómo están implicados los SCA en el ordenamiento espacio-temporal de las prácticas sociales en las organizaciones en que han laborado.

b) Conceptualizaciones de segundo orden sobre los SCA. Los investigadores contables también podrían teorizar a partir de los planteamientos o argumentos proporcionados por los contadores públicos y practicantes partícipes en un sistema de interacción específico. Estas elaboraciones serían de segundo orden, pues desde los planteamientos de Giddens las elaboraciones de primer orden son aquellas realizadas por el actor directamente involucrado en la acción social estudiada.

c) Perspectivas teóricas y metodológicas para el desarrollo de la investigación contable aplicada: El tipo de investigación contable sobresaliente en la literatura revisada para esta investigación es de carácter interpretativo, el cual busca principalmente, estudiar las prácticas contables en los contextos sociales en los que acontecen y cómo están implicadas en la constitución del mundo social. Específicamente las investigaciones que han utilizado la teoría de la estructuración como marco analítico, han sido clasificadas como de tipo interpretativo, tal como lo señala Rosendel R. (citado en Ryan, Scapens \& Theobald, 2004, 319), y a la vez han esquematizado las características generales de la investigación de tipo interpretativo, las cuales se sistematizan en la Tabla 2 .

El supuesto ontológico con respecto a la realidad social generalmente asumido en los artículos revisados en esta investigación, es acorde al que subyace en el tipo de investigación interpretativa, y es que la realidad es construida socialmente por los actores, los cuales usan modalidades para mediar entre las estructuras y su acción social, evidenciando que las prácticas sociales son actualizadas en las acciones de los individuos y producidas y reproducidas en el trasegar de la vida cotidiana. Así, el estudio de las prácticas sociales en un contexto específico, se torna relevante en tanto dichas prácticas pueden cambiar en cierto grado las estructuras sociales. 
Tabla 2. Investigación contable interpretativa ${ }^{10}$

\begin{abstract}
A. Creencias sobre el conocimiento
La teoría se usa para explicar las intenciones humanas. Su adecuación se valora por la consistencia lógica, la interpretación subjetiva y el acuerdo con las interpretaciones de sentido común de los actores.
\end{abstract}

\title{
B. Creencias sobre la realidad física y social
}

La realidad se crea socialmente y se objetiviza mediante la interacción humana. La acción humana es intencional y su significado se basa en el contexto histórico y social. Se asume el orden social y se media en los conflictos a través de los significados compartidos.

\section{Relación entre teoría y práctica contable}

La teoría contable busca explicar la acción y comprender cómo se produce y reproduce el orden social.

La clarificación del supuesto ontológico que subyace en cada investigación es vital para la escogencia de los instrumentos metodológicos con los cuales será abordada la investigación, pues podrían ser distintos cuando se percibe que la realidad existe per se por fuera de los individuos, así se reconoce la mediación de la subjetividad en la construcción de la realidad social.

En las investigaciones que utilizan la teoría de la estructuración para el estudio de los MAS, también ha sido generalizado el uso de los estudios de caso como método de investigación predilecto para examinar las prácticas contables-administrativas, los valores compartidos por los miembros organizacionales, las normas y reglas legitimadas en la organización, los recursos tanto de autoridad como de asignación implicados en los MAS, los significados subyacentes en la prácticas de los individuos, así como todas las posibles variables que podrían ser estudiadas en un sistema de interacción social.

En reiteradas ocasiones los investigadores contables argumentan que, más que buscar leyes generales que expliquen la realidad social sirviéndose de numerosos estudios de caso (en tanto constituyan una muestra representativa de la población objeto de estudio), lo que pretenden es la comprensión de las prácticas contables a través de la profundización que este tipo de estudios posibilitan. Éstos deben guiarse por un marco de referencia que permita trascender el nivel descriptivo y anecdótico para avanzar hacia la comprensión y explicación de la realidad social estudiada (Scapens, 1990, citado en Busco, 2000, p. 8).
"Desde una perspectiva interpretativa, las generalizaciones y leyes generales no explican, sólo las circunstancias específicas del caso se pueden usar para explicar. El sistema social particular en estudio y su contexto, proporcionan la base para una explicación. Son las relaciones entre diferentes partes del sistema y la propia relación del sistema con el sistema más grande del que forma parte (o sea, su contexto ampliado), lo que sirve para explicar el sistema" (Ryan, Scapens \& Theobald, 2004, 198).

Aunque los estudios de caso sean pertinentes para el progreso de la investigación interpretativa, presentan dos limitantes en cuanto a las posibilidades de su desarrollo y a sus resultados obtenidos. La primera limitante está relacionada con el uso reservado de la información contable, mientras la segunda se refiere a los procesos propios de desarrollo de las ciencias sociales en las que se vuelve impertinente extrapolar los resultados de investigación de un caso específico a contextos más generales.

Tal como ha sido señalado en esta investigación, la información contable se constituye en un recurso de autoridad del tipo inmaterial que ha ganado suma importancia en el sistema económico vigente. Esta información posibilita en ocasiones que las organizaciones obtengan ventajas competitivas en el mercado por el contenido que revela o por la naturaleza misma de los procesos que acontecen en ellas y que las diferencia de las demás. De esta manera, la investigación contable aplicada presenta una limitante en el momento que los investigado-

10 Adaptado de Chua W. F. (1986) por Ryan, B. Scapens, R. \& Theobald, M. Metodología de la investigación en Finanzas y Contabilidad. Barcelona: Ediciones Deusto $(2004,61)$. 
res requieran acceder a la información contable de una empresa específica. Este obstáculo podría ser trascendido poco a poco en la medida en que sea posible justificar que el estudio de caso de una organización específica esté centrado más en la compresión de las prácticas contables ahí acontecidas que en sus resultados, y valorar que dicho estudio podría aportar significativamente a la reflexividad de la vida cotidiana, anticipando los "traumas" propios de un cambio organizacional o colaborar en el manejo las consecuencias no esperadas de una transformación.

Otra limitante del progreso de la investigación interpretativa con respecto a los resultados obtenidos de los estudios de caso, es que éstos podrían circunscribirse a los contextos específicos en los cuales fueron desarrollados. Para la constitución de un programa de investigación sería necesario realizar estudios de caso en contextos nuevos o diferentes, con el fin ampliar las capacidades explicativas de la teoría sobre las distintas realidades estudiadas (Ryan, Scapens \& Theobald, 2004: 200-201).

Un punto adicional a tener en cuenta en el momento de desarrollar un estudio de caso, es la necesidad de escoger uno que sea adecuado para los intereses de la investigación. Guiado por la teoría de la estructuración un investigador podría estudiar cómo están constituidos los sistemas de responsabilidad en una organización y de qué manera la información contable influye en el comportamiento de los miembros organizacionales. Una investigación de este tipo requeriría como mínimo que la estructura de la organización objeto de estudio esté establecida por áreas de responsabilidad, desde las cuales la información contable sea relevante para evaluar el desempeño de los responsables de un área específica.

También sería importante tener en cuenta que, dada la disponibilidad de recursos, el acceso a la información y el conocimiento previo que se tenga de la organización, puede ser más pertinente realizar en primera instancia un estudio de caso de tipo exploratorio que identifique las variables que podrían ser estudiadas en posteriores estudios de caso con pretensiones explicativas.

Un aspecto adicional, que también podría resultar importante en el desarrollo de los estudios de caso, sería definir el tipo de función que asumiría el investigador en el proceso de investigación, en donde podría intervenir como: extraño, visitante, facilitador, participante o actor (Ryan, Scapens \& Theobald, 2004: 204- 205).

Ahora bien, teniendo en cuenta los escenarios posibles para el desarrollo de un programa matricial de investigación estructuracionista, que busca explicar a través de estudios de caso orientados por un marco previamente elaborado acerca de las prácticas contables en los contextos específicos en que se desarrollan, para lo cual resultaría importante indicar el itinerario que podría acogerse en el momento de constituir la metodología para su tratamiento.

\subsection{Itinerario metodológico}

Después de clarificar los supuestos ontológicos frente a la realidad que pueden ser compartidos o discutidos por los miembros de la posible comunidad científica a constituir, es necesario acordar entre otras, las siguientes pautas metodológicas a tener en cuenta en el desarrollo de las investigaciones planteadas por los miembros de la comunidad:

a) Definir el problema de investigación, el estado de avance de la temática y los límites y alcances de la investigación. Esta delimitación estará más demarcada en la medida que el programa de investigación se consolide, dado que en este proceso será necesario conocer qué avances conceptuales y trabajos empíricos han sido desarrollados por los miembros de la comunidad.

b) Identificar los datos necesarios y las fuentes de información. En algunos casos en los cuales se requiera estudiar los SCA desde las dimensiones de la teoría estructuración, es importante reflexionar acerca de que las estructuras de significado, legitimación y dominación, de ser consideradas como variables, no podrían ser observables empíricamente, lo cual debe ser tenido en cuenta en el momento de definir los instrumentos metodológicos con los cuales pretendieran ser captados.

c) Diseñar la metodología, el tipo de estudio a realizar y los instrumentos con los cuales serán recogidos los datos. Para el 
avance del programa de investigación, sería necesaria la socialización del tipo de instrumentos y herramientas utilizadas por los investigadores contables administrativos que ya han experimentado la realización de investigaciones, tanto teóricas como empíricas.

d) Tratamiento y sistematización de la información colectada. Dado que en la investigación contable de tipo interpretativa, se reconoce el papel activo del sujeto en la construcción del conocimiento, será necesaria la constitución ordenada de un archivo que permita la triangulación de la información obtenida de diversas fuentes y los procesos llevados a cabo en la sistematización de la información para hacer más viable la demostración de la validez de los resultados en el caso que sea requerida.

e) Análisis a partir de la información colectada. En este punto es importante indicar que los procesos de escritura de los investigadores contables deben evidenciar que las interpretaciones realizadas, corresponde efectivamente a los hallazgos de investigación orientados por los marcos escogidos para el análisis. En el caso de trabajos que se enfoquen más al desarrollo conceptual, también será necesario explicitar las posibles redes teóricas que se han establecido o que se pueden establecer al interior del programa de investigación.

Después de indicar los posibles indicios de un programa de investigación estructuracionista, vinculado al estudio de los SCA, sus escenarios posibles y el itinerario metodológico que podría seguirse en la realización de una investigación dentro del programa, es necesario resaltar finalmente que los programas de investigación son construcciones sociales que se consolidan y mantienen en el tiempo en la medida que exista una tradición escrita que los sustente y una comunidad científica que comparta las teorías, valores, instrumentos metodológicos y posibles soluciones, y generen unos modelos que logren guiar las futuras investigaciones.

\section{Conclusiones}

La forma como los individuos experimentan el espacio-tiempo en la vida social moderna demarca un carácter distintivo de la modernidad con respecto a los órdenes sociales que le anteceden. Bajo la modernidad las relaciones sociales han sido reancladas espacio-temporalmente en diversos sistemas de interacción, uno de éstos son los SCA, los cuales están localizados en otros sistemas de interacción más amplios como lo son las organizaciones, las cuales a su vez surgen y pueden ser estudiadas en un contexto aún más amplio como lo es la vida social moderna.

La teoría de la estructuración de Anthony Giddens, es aquí analizada como un proyecto conceptual, desde el que se imparte orden y se da forma a procesos que indagan sobre la vida social desde distintas ciencias y disciplinas; pero especialmente a partir del estudio sociológico. Dicho proyecto logra dar luz a la interpretación y explicación de aspectos de material importancia referentes a la conducta humana y a los procesos y modos de constitución de la sociedad moderna. Así se contribuye a la tarea de definir generalizaciones válidas para el estudio de la vida social, atendiendo al tiempo, varios otros propósitos también prioritarios en ciencias sociales. A partir del análisis de la teoría así concebida como proyecto conceptual, se sistematizan las que aquí se denominan categorías conceptuales o analíticas de Giddens, correspondientes a términos que cobran un significado preciso en la teoría de la estructuración.

Concibiendo los SCA como el diseño de relaciones sociales ubicadas espacio-temporalmente en el nivel de las interacciones, definimos los mismos como el orden virtual de prácticas sociales ordenadas de manera reflexiva, que son producidas y reproducidas por los agentes en las organizaciones modernas, a través de contextos regularizados de interacción. Los SCA en las organizaciones, dan lugar a contextos sociales estructurantes, en tanto delimitan sectores de espacio-tiempo en los cuales se ejecutan ciertas prácticas y se propician ciertos encuentros sociales entre los agentes.

$\mathrm{Al}$ entender los SCA como sistemas de interacción que expresan las relaciones sociales existentes, donde los agentes participan en su producción y reproducción, es necesario estudiar cuál ha sido su papel en los cambios sociales y organizacionales, generando así una conciencia del poder de transfor- 
mación de las estructuras sociales que tanto la Contabilidad entendida como esquema de comprensión y modalidad, como los procesos que ésta propicia a partir de cada uno de los actos involucrados en los SCA, desencadenan en el mantenimiento y cambio de las prácticas sociales institucionalizadas.

La teoría de la estructuración ofrece sin duda un amplio potencial en la tarea de exploración que compete a la investigación contable-administrativa, que se interese por una orientación sociológica; potencial éste, constituido por las nuevas oportunidades que la conformación de un programa de investigación alrededor de esta temática podría significar en general para la Contabilidad en materia teórica y metodológica, y por las posibilidades que su recategorización al caso específico de las organizaciones de América Latina y del país, traería en la comprensión de los SCA que competen a nuestro contexto.

El análisis que aquí se hace de los SCA desde la teoría de la estructuración, permite visualizar algunas potencialidades que surgen en la investigación contable-administrativa, al utilizar este marco de interpretación. Así, nos aventuramos a plantear finalmente que puede ser posible construir un programa de investigación específicamente en contabilidad, desde el cual se trabaje cómo se han constituido los SCA en las organizaciones del país, utilizando estudios de caso y otros tipos de metodologías tanto de corte teórico, como de aplicación empírica, para recategorizar los conceptos construidos a partir de esta teoría, con el fin de diseñar sistemas de administración y de gestión contable, más acordes a la realidad de nuestro contexto, desde los cuales se pueda hacer una lectura más amplia de las prácticas sociales que se presentan en este ámbito.

\section{Referencias}

Aktouf, O. (2000) La metodología de las ciencias sociales y el enfoque cualitativo en las organizaciones. Una introducción al procedimiento clásico y una crítica. (Traducido por: Sánchez, K.). Artes gráficas del Valle: Cali.

Araujo, P. (2003) Nuevo Institucionalismo, Teoría de la Estructuración y cambio en los sistemas y prácticas de contabilidad de gestión: teorías y métodos de investigación. En: Revista Española de Financiación y Contabilidad. 32: 118, 693-724.

Beriain, J. (1996) Consecuencias perversas de la modernidad, (Comp.). Anthropos: Barcelona.
Boland, R. (1996) Why shared meanings have no place in structuration theory: A reply to Scapens and Macintosh. En: Accounting, Organization and Society. Gran Bretaña: Elsevier Science Ltd. 21: 691-697.

Burns, J. \& Scapens, R. (2000) Conceptualizing management accounting change: an institutional framework. En: Management Accounting Research. 11: 3-25.

Busco, C, Riccaboni, A \& Scapens, R. (2000) Management Accounting Systems and Organisational Culture: an Institutional Framework for Interpreting their Linkages and Processes of Change. Paper prepared for the 2nd workshops on Management Accounting Change. 11-14: 43.

Carmona, S, Ezzamel, M, \& Gutiérrez, F. (2002). The relationship between accounting and spatial practices in the factory. En: Accounting, Organizations and Society. Gran Bretaña: Elsevier Science Ltd. 27: 239-274.

Englund, H \& Gerdin, J. (2007). "Structuration theory and mediating concepts: Pitfalls and implications for management accounting research" Critical perspectives on accounting. Gran Bretaña: Elsevier Science. pp. 1-13. Tomado de: www. elsevier.com/locate/cpa.

Giddens, A. (1998). La constitución de la sociedad. Bases para la teoría de la estructuración. (Traducido por: José Luis Echeverry). Amorrortu: Buenos Aires.

Giddens, A. (1999). Consecuencias de la modernidad. (Traducido por: Ana Lizón Ramón). Alianza Editorial: Madrid.

Klir, G. (1978) Tendencias en la teoría general de sistemas, (Comp). (Traducido por: Álvaro Delgado y Andrés Ortega) Alianza Editorial : Madrid.

Kuhn, T. (2000) La estructura de las revoluciones científicas. Bogotá : Fondo de Cultura Económica.

Lawrence, S. Alam, M, Northcott, D. \& Lowe, T. (1997). Accounting systems and systems of accountability in the New Zealand health sector. En: Accounting, Auditing \& Accountability Journal. 10: 665-683.

Lakatos, I. (2007) La metodología de los programas de investigación científica. (Traducido por: Juan Carlos Zapatero). Alianza Editorial: Madrid.

Macintosh, N \& Scapens, R. (1990) Structuration Theory in Management Accounting. En: Accounting, Organizations and Society. Gran Bretaña: Pergamon Press plc. 15: 455-477.

Macintosh, N \& Scapens, R. (1996) Structure and agency in management accounting: a response to Boland's interpretive act. En: Accounting, Organizations and Society. Gran Bretaña : Elsevier Science. 21: 7-8.

Macintosh, N \& Scapens, R. (1991) Management Accounting and Control Systems: A Structuration Theory- Analysis. En: Journal of Management Accounting Research. 3: 131 - 158.

Matthew R. \& Karsten, H (2008) Review: Giddens's Structuration theory and information Systems research. En: MIS Quarterly, $32: 1$

Mayntz, R. (1972) Sociología de la organización. Madrid: Alianza editorial.

Roberts, J \& Scapens, R. (1985) Accounting systems and systems of accountability - Understanding accounting practices in their organizational contexts. En: Accounting, Organizations and Society. 10: 443-456.

Ryan, B, Scapens, R. \& Theobald, M. (2004). Metodología de la investigación en Finanzas y Contabilidad. Barcelona: Deusto. 